\title{
Diminished accuracy of biomarkers of fibrosis in low replicative chronic hepatitis B
}

\author{
Faisal M. Sanai ${ }^{1,8^{*}}$, Taha Farah ${ }^{8}$, Khalid Albeladi ${ }^{8}$, Faisal Batwa ${ }^{8}$, Yaser Dahlan ${ }^{8}$, Mohammed A. Babatin ${ }^{3}$, \\ Hamad Al-Ashgar ${ }^{4}$, Hadeel AlMana ${ }^{5}$, Khaled S. Alsaad ${ }^{6}$, Khalid AlSwat ${ }^{1}$, Abdulrahman Aljumah ${ }^{7,9}$, \\ Ibrahim H. AlTraif ${ }^{7}$, , Bahaa E. Kailani $^{10}$ and Khalid I. Bzeizi ${ }^{2}$
}

\begin{abstract}
Background: We evaluated the diagnostic accuracy of aspartate aminotransferase (AST)-to-platelet ratio index (APRI), fibrosis-4 index (FIB-4), AST/alanine aminotransferase (ALT) ratio (AAR), and age-platelet index (API) for significant fibrosis (Metavir F2-4) in low-replicative (HBV DNA $<20,000 \mathrm{IU} / \mathrm{mL}$ ) chronic hepatitis B virus (HBV) patients.
\end{abstract}

Methods: The sensitivity, specificity, and area under the receiver-operating characteristic curve (AUROC) of HBeAgnegative, low-replicative ( $n=213$ ) and high-replicative (HBV DNA $\geq 20,000 \mathrm{IU} / \mathrm{mL}, n=153$ ) patients was assessed.

Results: Overall, 113 patients (30.9\%) had F2-4 fibrosis. Of the low and high-replicative patients, 40 (18.8\%) and 73 (47.7\%) had F2 -4 , respectively $(P<0.0001)$. APRI $\geq 0.5$ less frequently identified F2-4 fibrosis in low vs. highreplicative patients $(48.7 \%$ vs. $69.6 \%, P=0.032)$ and AAR identified it more frequently in low-replicative patients (37. $5 \%$ vs. $19.4 \%, P=0.037)$. FIB-4 and API were not different $(P>0.05)$ for identifying F2-4 fibrosis in low and highreplicative patients. Higher specificities were seen at the lowest cut-offs in low vs. high-replicative states for APRI $(\geq 0.5,98 \%$ vs. $68.9 \%)$, AAR (84.3\% vs. $76.6 \%)$, FIB-4 ( $\geq 1.45,97.5 \%$ vs. $87.8 \%)$ and API ( $>4,94.8 \%$ vs. $93.8 \%)$. At ROCdefined thresholds, APRI $(\geq 0.33)$, AAR $(\geq 0.93)$, FIB-4 $(\geq 0.70)$ and API $(>2)$ showed greater AUROCs for F2-4 diagnosis in low replicative $(0.80,0.62,0.81$ and 0.71 , respectively) vs. high-replicative patients $(0.73,0.52,0.67$ and 0.69 , respectively).

Conclusion: All 4 biomarkers in both, low and high-replicative HBV demonstrate modest accuracy for fibrosis diagnosis at conventional cut-offs. Lowering the cut-offs may increase the diagnostic relevance of these biomarkers, particularly for APRI and FIB-4 in low-replicative disease.

Keywords: Hepatitis B, Fibrosis, Biomarkers, APRI

\section{Background}

Assessment of histological disease is of immense importance in managing chronic hepatitis B virus (HBV) patients. Treatment is strongly recommended in patients with significant fibrosis (for instance, METAVIR score F2-4). In the absence of liver biopsy, treatment decisions are based on the surrogate markers of histological

\footnotetext{
* Correspondence: sanaifa@ngha.med.sa

${ }^{1}$ Liver Disease Research Center, King Saud University, Riyadh, Saudi Arabia ${ }^{8}$ Gastroenterology Unit, Department of Medicine, King Abdulaziz Medical City, PO Box: 9515, Jeddah 21423, Saudi Arabia

Full list of author information is available at the end of the article
}

disease, namely, elevated alanine aminotransferase (ALT) and high HBV DNA levels [1,2].

Significant fibrosis in patients with low viremia may occur, with reports of progression to cirrhosis [3]. We have previously shown that F2-4 fibrosis occurs in about $18 \%$ of patients with HBV DNA $<20,000 \mathrm{IU} / \mathrm{mL}$ [4]. Thus, since fibrosis occurs in a minority of patients with 'low' viremia levels, it becomes essential to identify its predictors, prior to undertaking liver biopsy. Furthermore, a single biopsy does not measure the dynamic nature of liver fibrosis [5]. 
Of the several combined biomarkers of fibrosis, FIB-4 (based on age, ALT, aspartate aminotransferase [AST] and platelet count), AST-to-platelet ratio index (APRI), AST/ALT ratio (AAR), and age-platelet index (API) have been well studied. Studies have suggested that these markers may be useful for predicting fibrosis and cirrhosis [6]. However, most validations occurred under controlled conditions and have shown sensitivity and specificity at the extreme stages of liver fibrosis. Additionally, previous studies have not evaluated these combination biomarkers in the context of low HBV DNA levels.

In this study, we aimed to evaluate the diagnostic performance of serum biomarkers in identifying significant fibrosis in an unselected cohort of HBeAg-negative, lowreplicative, chronic hepatitis $\mathrm{B}$ patients.

\section{Methods}

\section{Study patients}

Consecutive patients were included by a retrospective search of clinical records and hospital databases for the period between January 2006 and January 2012. All data was anonymized and de-identified prior to analysis. The patients were included from four centres in Saudi Arabia. The institutional review boards of all centres (Retrospective Research Committee, Institutional Review Board, King Abdullah International Medical Research Centre; Research Advisory Council, King Faisal Specialist Hospital and Research Centre; Research and Ethics Committee, Prince Sultan Military Medical City; Central Institutional Review Board; Ministry of Health) approved the study. Eligible patients for the analysis had detectable HBsAg $>6$ months prior to inclusion in the study. All patients were $\mathrm{HBeAg-negative} \mathrm{and} \mathrm{were} \mathrm{between} 16$ to 80 years of age.

The exclusion criteria were: (i) co-infection with hepatitis C, delta virus or HIV; (ii) superimposed with other liver diseases; (iii) hepatotoxic medications in the preceding 3 months; (iv) previous immunosuppressive or antiviral therapy; (v) decompensated cirrhosis with a Child-Pugh score $>6$, or evidence of portal hypertension, variceal bleeding, laboratory findings of a platelet count $<100\left(10^{9} / \mathrm{L}\right)$, an international normalized ratio $\geq 1.3$; (vii) creatinine $>135 \mu \mathrm{mol} / \mathrm{L}$ (viii) presence of hepato-biliary malignancy; (ix) alcohol consumption $>20$ g/day; and (x) organ transplantation.

\section{Study design}

A minimum of three recordings of liver biochemistry for patients with normal levels and two recordings for those with elevated levels were required. The median number of ALT tests performed pre liver biopsy was 5 (interquartile range [IQR] 4-8), and these were sampled over a median period of 21 months (IQR 8-46). Serum biochemical and haematological values prior to the liver biopsy were recorded. Routine liver biochemical tests were performed using commercially available autoanalysers and hepatitis serological markers were assayed using commercially available enzyme-linked immunoassays. Patients with HBV DNA $<20,000 \mathrm{IU} / \mathrm{mL}$ were classified as lowreplicative while those with $\mathrm{HBV}$ DNA $\geq 20,000 \mathrm{IU} / \mathrm{mL}$ were classified as high-replicative. A minimum of three HBV DNA recordings was required and the reference value utilized for analysis was based on the pre biopsy level. Serum HBV DNA level was expressed in $\mathrm{IU} / \mathrm{mL}$ (1 IU $/ \mathrm{mL}=5.6$ copies $/ \mathrm{mL}$ ) [7]. Quantitative HBV DNA levels were measured by a COBAS TaqMan System (Roche Diagnostics, Indianapolis, IN, USA), which has a lower detection limit of $15 \mathrm{IU} / \mathrm{mL}$, or Abbott Real-Time HBV assay (Abbott Molecular, Inc., Des Plaines, IL, USA), with a lower detection limit of $10 \mathrm{IU} / \mathrm{mL}$. The median duration between the first and pre liver biopsy HBV DNA was 12 months (IQR 6-20).

\section{Measurement of noninvasive biomarkers}

Patient data included age (at time of liver biopsy), ALT and AST levels (upper limit of normal [ULN] values that were specific to the laboratory performing the test) and platelet counts. APRI, FIB-4 and AST/ALT ratio were calculated based on laboratory results from sera collected within 3 months of the liver biopsy using the following formulas:

$$
\begin{aligned}
\text { FIB- } 4= & \frac{\text { Age }(\text { years }) \times \operatorname{AST}(\mathrm{U} / \mathrm{L})}{\text { Platelet count }\left(10^{9} / \mathrm{L}\right) \times[\operatorname{ALT}(\mathrm{U} / \mathrm{L})]^{1 / 2}} \\
\mathrm{AAR} & =\operatorname{AST} / \operatorname{ALT} \\
\mathrm{APRI} & =\frac{\operatorname{AST}(/ \mathrm{ULN})}{\text { Platelet count }\left(10^{9} / \mathrm{L}\right)} \times 100
\end{aligned}
$$

API: Age (years): $<30=0 ; 30-39=1 ; 40-49=2 ; 50-$ $59=3 ; 60-69=4 ; \geq 70=5$. Platelet count: $>225=0$; $200-224=1 ; 175-199=2 ; 150-174=3 ; 125-149=4$; $<125=5$. AP index is the sum of the above (possible value $0-10)$.

\section{Liver histology}

All patients included in this study had liver biopsies stained with hematoxylin and eosin for morphological evaluation and reticulin for the assessment of fibrosis. Only liver specimens with more than nine portal tracts were considered sufficient. All specimens were centrally assessed and scored according to the METAVIR scoring system [8] by two experienced hepatopathologists who were blinded to all clinical information. Fibrosis scores of F0-1 were defined as minimal/mild, F2-4 as significant. 


\section{Statistical analyses}

Quantitative variables were expressed as the mean \pm standard deviation, and categorical variables as frequencies and proportions. The unpaired student's t-test was used to compare between the means or log means of the variable if the variable was normally or not normally distributed, respectively. The Chi-square or Fishers' exact tests were used to compare frequencies and proportions in categorical variables, as appropriate. The sensitivity, specificity, positive likelihood ratio ( $\mathrm{LR}+$ ), and negative likelihood ratio (LR-) of points on curve were calculated to obtain the optimal cut-off point to discriminate F2-4 with noninvasive biomarkers. The area under receiver operating characteristics (AUROC) curve was used to assess the overall diagnostic value of the biomarkers at different cut-offs that predicted fibrosis. All of the tests of significance were 2 -tailed and a $P$-value of $<0.05$ was considered statistically significant. Statistical Package for Social Sciences (SPSS, version 17.0; Chicago, IL, USA) and MedCalc (MedCalc Software, Inc., Mariakerke, Belgium) were used for data analysis.

\section{Results}

\section{Baseline characteristics of patients}

A total of 366 patients were included in this analysis and of these 213 (58.2\%) had HBV DNA < 20,000 IU/mL, and $153(41.8 \%)$ had HBV DNA $\geq 20,000 \mathrm{IU} / \mathrm{mL}$. The mean age was $38.6 \pm 11.8$ years and males constituted 272 (74.3\%). In the overall cohort, F2-4 fibrosis was seen in $113(30.9 \%)$ patients of whom 20 (5.5\%) had F4 fibrosis. Of the low-replicative (HBV DNA $<20,000 \mathrm{IU} / \mathrm{mL}$ ) and highreplicative $(\geq 20,000 \mathrm{IU} / \mathrm{mL})$ patients, $40(18.8 \%)$ and 73 $(47.7 \%)$ had F2-4, respectively $(P<0.0001$, Table 1$)$. Low replicative patients were more obese compared to highreplicative ones $(P=0.002)$ and more likely to have normal ALT (55.4\% vs. $19.6 \%, P<0.0001)$. Mean biomarker scores between low-replicative and high-replicative patients were not significantly different $(P>0.05)$, however the mean scores were significantly higher for all biomarkers for F2-4 (compared to F0-1, $P<0.05$, data not shown). Furthermore, all four biomarkers more frequently identified F2-4 fibrosis in both, low and high-replicative patients $(P<0.05$, Additional file 1: Table S1).

Overall, there was an association between fibrosis stage and APRI, FIB- 4 and API $(P<0.05$ for all). For each successive fibrosis stage from F0 to F4, significantly higher APRI, FIB- 4 and API values were seen $(P<0.0001$ for all, Table 2). There was no difference between successive fibrosis stages by AAR $(P=0.445)$.

\section{Sensitivity and specificity of biomarkers in relation to HBV DNA levels}

For high-replicative patients, an APRI $\geq 0.5$ more frequently identified F2-4 fibrosis $(69.6 \%)$ as compared to those with low-replication $(48.7 \%, P=0.032)$. Other APRI cut-offs were not significantly different between the two groups (Table 3). On the other hand, AAR $>1.0$ less frequently identified F2-4 fibrosis in high-replicative patients (19.4 vs. $37.5 \%, P=0.037)$. None of the cut-offs for FIB-4 or API differentiated F2-4 vs. F0-1 fibrosis between low and high-replicative patients. The specificity for F2-4 fibrosis at the lowest cut-offs was consistently greater in low compared to high-replicative states for APRI (98.0\% vs. $68.9 \%$, respectively), AAR ( $84.3 \%$ vs. $76.6 \%$, respectively), FIB-4 (97.5 vs. $87.8 \%$, respectively) and API (94.8\% vs. $93.8 \%$, respectively). This pattern remained similar for higher cut-offs (Table 4).

\section{Performance characteristics of biomarkers}

The overall sensitivity, specificity, LR+, LR- and AUROCs of the four biomarkers at different cut-offs for the diagnosis of F2-4 fibrosis are shown in Additional file 2:

Table 1 Baseline Characteristics of patients in relation to HBV DNA levels

\begin{tabular}{|c|c|c|c|}
\hline Parameters & $\begin{array}{l}\text { HBV DNA }<20,000 \mathrm{IU} / \mathrm{mL} \\
(n=213)\end{array}$ & $\begin{array}{l}\text { HBV DNA }>20,000 \mathrm{IU} / \mathrm{mL} \\
(n=153)\end{array}$ & $P$ value \\
\hline Age (years) & $38.2 \pm 11.7$ & $39.1 \pm 12.0$ & 0.485 \\
\hline Male gender (\%) & $156(73.2)$ & $116(75.8)$ & 0.578 \\
\hline $\mathrm{BMI}\left(\mathrm{Kg} / \mathrm{m}^{2}\right)$ & $29.5 \pm 10.4$ & $26.9 \pm 4.9$ & 0.002 \\
\hline Diabetes mellitus (\%) & $30(14.1)$ & $20(13.1)$ & 0.781 \\
\hline Hyperlipidemia (\%) & $41(19.2)$ & $31(20.3)$ & 0.810 \\
\hline HBV DNA $\log _{10}$ & $3.3 \pm 0.7$ & $6.1 \pm 1.3$ & $<0.0001$ \\
\hline Platelets $\left(10^{9}\right)$ & $255.3 \pm 68.9$ & $232.2 \pm 61.8$ & 0.001 \\
\hline Elevated AST (\%) & $41(20.0)$ & $80(54.1)$ & $<0.0001$ \\
\hline Normal ALT (\%) & $118(55.4)$ & 30 (19.6) & $<0.0001$ \\
\hline Elevated ALT (\%) & $95(44.6)$ & $123(80.4)$ & \\
\hline Fibrosis (F2-4, \%) & $40(18.8)$ & $73(47.7)$ & $<0.0001$ \\
\hline
\end{tabular}

Data expressed as mean \pm standard deviation or $\mathrm{n}(\%)$ as appropriate. $\mathrm{n}$, number. $A S T$ aspartate aminotransferase, $A L T$ alanine aminotransferase ratio, BMI body mass index, $H B V$ hepatitis $B$ virus 
Table 2 Mean scores of the four biomarkers in increasing stages (Metavir) of liver fibrosis

\begin{tabular}{|c|c|c|c|c|c|c|c|c|}
\hline Fibrosis Stage & APRI $(95 \% \mathrm{Cl})$ & $P$ value & AAR $(95 \% \mathrm{CI})$ & $P$ value & FIB-4 (95\% Cl) & $P$ value & API $(95 \% \mathrm{Cl})$ & $P$ value \\
\hline STAGE 0 & $\begin{array}{l}0.32 \\
(0.27-0.38)\end{array}$ & $<0.0001$ & $\begin{array}{l}0.77 \\
(0.31-0.42)\end{array}$ & 0.445 & $\begin{array}{l}0.66 \\
(0.54-0.78)\end{array}$ & $<0.0001$ & $\begin{array}{l}1.58 \\
(1.22-1.94)\end{array}$ & $<0.0001$ \\
\hline STAGE 1 & $\begin{array}{l}0.38 \\
(0.31-0.45)\end{array}$ & & $\begin{array}{l}0.65 \\
(0.69-0.83)\end{array}$ & & $\begin{array}{l}0.71 \\
(0.63-0.78)\end{array}$ & & $\begin{array}{l}1.74 \\
(1.52-1.96)\end{array}$ & \\
\hline STAGE 2 & $\begin{array}{l}0.71 \\
(0.52-0.90)\end{array}$ & & $\begin{array}{l}0.68 \\
(0.60-0.77)\end{array}$ & & $\begin{array}{l}1.08 \\
(0.88-1.29)\end{array}$ & & $\begin{array}{l}2.59 \\
(2.11-3.07)\end{array}$ & \\
\hline STAGE 3 & $\begin{array}{l}0.88 \\
(0.66-1.09)\end{array}$ & & $\begin{array}{l}0.86 \\
(0.58-1.14)\end{array}$ & & $\begin{array}{l}1.66 \\
(1.06-2.25)\end{array}$ & & $\begin{array}{l}3.56 \\
(2.68-4.44)\end{array}$ & \\
\hline STAGE 4 & $\begin{array}{l}1.13 \\
(0.51-1.76)\end{array}$ & & $\begin{array}{l}0.96 \\
(0.68-1.24)\end{array}$ & & $\begin{array}{l}2.52 \\
(1.49-3.55)\end{array}$ & & $\begin{array}{l}5.10 \\
(3.93-6.27)\end{array}$ & \\
\hline
\end{tabular}

AST aspartate aminotransferase, APRI AST-to-platelet ratio index, AAR AST/alanine aminotransferase ratio, API age-platelet index

Table S2. APRI demonstrated similar AUROCs for low and high-replicative states at all cut-offs (Table 4). However, higher AUROCs for F2-4 diagnosis were observed for low- compared to high-replicative state with AAR (0.61 vs. 0.48), FIB-4 (0.67 vs. 0.59) and API (0.66 vs. 0.55). Additionally, the AUROCs were serially lower across all biomarkers for both low and high-replicative states with incremental increase in the cut-off levels.

Figure 1a depicts the ROC curve analysis for optimum cut-offs with the best compromise sensitivity-specificity for distinguishing F2-4 for the four biomarkers in lowreplicative state. At the ROC-derived optimum cut-offs, the sensitivity for APRI $(\geq 0.33)$ is $76.4 \%$ and the specificity is 74.4\% (AUROC, 0.80; 95\% CI: 0.73-0.85; $P<0.0001)$, for AAR $(\geq 0.93)$ the sensitivity is $73.5 \%$ and the specificity 47.5\% (AUROC, 0.62; 95\% CI: 0.55-0.69; $P=0.023)$, for FIB-4 $(\geq 0.70)$ the sensitivity is $72.9 \%$ and specificity is $76.9 \%$ (AUROC, 0.81 ; 95\% CI: 0.75-0.86; $P<0.0001)$, and for API $(>2)$ the sensitivity is $75.1 \%$ and specificity is $55.0 \%$ (AUROC, 0.71; 95\% CI: 0.64-0.77; $P<0.0001)$. Figure $1 \mathrm{~b}$ depicts the ROC curve analysis in high-replicative patients. In patients meeting these optimum cut-offs, the sensitivity for APRI $(\geq 0.33)$ is $52.7 \%$ and the specificity is $87.0 \%$ (AUROC, 0.73 ; 95\% CI: $0.65-$ $0.80 ; P<0.0001)$, for AAR $(\geq 0.91)$ the sensitivity is $24.7 \%$ and the specificity $79.2 \%$ (AUROC, 0.52 ; 95\% CI: $0.44-$ $0.60 ; P=0.643)$, for FIB-4 $(\geq 0.70)$ the sensitivity is $58.1 \%$ and specificity is $66.7 \%$ (AUROC, 0.67 ; 95\% CI: $0.59-0.75$; $P=0.0001)$, and for API $(>2)$ the sensitivity is $75.0 \%$ and specificity is 56.2\% (AUROC, 0.69; 95\% CI: 0.61-0.76; $P<0.0001)$.

Table 3 Diagnosis of significant fibrosis (F2-4) in low and high replication HBV patients based on the four biomarkers

\begin{tabular}{|c|c|c|c|c|}
\hline Fibrosis Biomarker & $\begin{array}{l}\text { Total } \\
(n=113)\end{array}$ & $\begin{array}{l}\text { HBV DNA }<20,000 \mathrm{IU} / \mathrm{mL} \\
(n=40)\end{array}$ & $\begin{array}{l}\text { HBV DNA > 20,000 IU/mL } \\
(n=73)\end{array}$ & $P$ value \\
\hline \multicolumn{5}{|c|}{ AST-Platelet Ratio Index (APRI) } \\
\hline$\geq 0.5$ & $67(62.0)$ & $19(48.7)$ & $48(69.6)$ & 0.032 \\
\hline$\geq 0.7$ & $46(42.6)$ & $13(32.3)$ & $33(47.8)$ & 0.143 \\
\hline$\geq 1.0$ & $26(24.1)$ & $7(17.9)$ & $19(27.5)$ & 0.263 \\
\hline$\geq 1.5$ & $11(10.2)$ & $2(5.1)$ & $9(13.0)$ & 0.191 \\
\hline \multicolumn{5}{|l|}{ AST/ALT Ratio (AAR) } \\
\hline$>1.0$ & $29(25.9)$ & $15(37.5)$ & $14(19.4)$ & 0.037 \\
\hline \multicolumn{5}{|l|}{ FIB-4 } \\
\hline$\geq 1.45$ & $35(32.4)$ & $14(35.9)$ & $21(30.4)$ & 0.560 \\
\hline$\geq 2.0$ & $25(23.1)$ & $9(23.1)$ & $16(23.2)$ & 0.999 \\
\hline$\geq 3.25$ & $10(9.3)$ & $6(15.4)$ & $4(5.8)$ & 0.164 \\
\hline \multicolumn{5}{|c|}{ Age-Platelet Index (API) } \\
\hline$>4$ & $34(30.1)$ & $15(37.5)$ & $19(26.0)$ & 0.204 \\
\hline$>5$ & $21(8.6)$ & $9(22.5)$ & $12(6.4)$ & 0.428 \\
\hline$>6$ & $12(10.6)$ & $6(15.0)$ & $6(8.2)$ & 0.263 \\
\hline$>7$ & $5(4.4)$ & $3(7.5)$ & $2(2.7)$ & 0.344 \\
\hline
\end{tabular}

Data expressed as $\mathrm{n}(\%) . N$ number, AST aspartate aminotransferase, ALT alanine aminotransferase ratio 
Table 4 Sensitivity, specificity and AUROCs of the four different biomarkers in patients with low and high-replicative HBV at different cutoff values in identifying significant fibrosis (F2-4)

\begin{tabular}{|c|c|c|c|c|c|c|}
\hline \multirow{2}{*}{$\begin{array}{l}\text { Biomarker } \\
\text { Cut-off }\end{array}$} & \multicolumn{3}{|c|}{$\mathrm{HBV}$ DNA $\leq 20,000 \mathrm{IU} / \mathrm{mL}$} & \multicolumn{3}{|c|}{$\mathrm{HBV}$ DNA $\geq 20,000 \mathrm{IU} / \mathrm{mL}$} \\
\hline & $\begin{array}{l}\text { Sensitivity } \\
(\%, 95 \% \mathrm{Cl})\end{array}$ & $\begin{array}{l}\text { Specificity } \\
(\%, 95 \% \mathrm{Cl})\end{array}$ & $\begin{array}{l}\text { AUROC } \\
(\%, 95 \% \mathrm{Cl})\end{array}$ & $\begin{array}{l}\text { Sensitivity } \\
(\%, 95 \% \mathrm{CI})\end{array}$ & $\begin{array}{l}\text { Specificity } \\
(\%, 95 \% \mathrm{Cl})\end{array}$ & $\begin{array}{l}\text { AUROC } \\
(\%, 95 \% \mathrm{Cl})\end{array}$ \\
\hline & \multicolumn{3}{|c|}{ AST-Platelet Ratio Index (APRI) } & \multicolumn{3}{|c|}{ AST-Platelet Ratio Index (APRI) } \\
\hline$\geq 0.5$ & $48.7(32.4-65.2)$ & $98.0(91.2-99.5)$ & $0.70(0.63-0.77)$ & $69.6(57.3-80.1)$ & $68.9(57.1-79.2)$ & $0.69(0.61-0.77)$ \\
\hline$\geq 0.7$ & $33.3(19.1-50.2)$ & $98.1(94.5-99.6)$ & $0.66(0.59-0.72)$ & $47.8(35.7-60.2)$ & $77.0(65.8-86.0)$ & $0.62(0.54-0.70)$ \\
\hline$\geq 1.0$ & $17.9(7.5-33.5)^{\mathrm{a}}$ & $99.4(96.5-100.0)$ & $0.59(0.51-0.66)$ & $27.5(17.5-39.6)$ & $91.9(83.2-97.0)$ & $0.60(0.51-0.68)$ \\
\hline \multirow[t]{2}{*}{$\geq 1.5$} & $5.1(0.6-17.3)^{\mathrm{a}}$ & $100.0(97.7-100.0)$ & $0.53(0.45-0.60)$ & $13.0(6.1-23.3)^{\mathrm{a}}$ & $95.9(88.6-99.2)$ & $0.54(0.46-0.63)$ \\
\hline & \multicolumn{3}{|c|}{ AST/ALT Ratio (AAR) } & \multicolumn{3}{|c|}{ AST/ALT Ratio (AAR) } \\
\hline \multirow[t]{2}{*}{$>1.0$} & $37.5(22.7-54.2)$ & $84.3(77.9-89.5)$ & $0.61(0.54-0.68)$ & $19.4(11.1-30.5)$ & $76.6(65.6-85.5)$ & $0.48(0.40-0.56)$ \\
\hline & \multicolumn{3}{|l|}{ FIB-4 } & \multicolumn{3}{|l|}{ FIB-4 } \\
\hline$\geq 1.45$ & $35.9(21.2-52.8)$ & $97.5(93.7-99.3)$ & $0.67(0.60-0.73)$ & $30.4(19.9-42.7)$ & $87.8(78.2-94.3)$ & $0.59(0.51-0.67)$ \\
\hline$\geq 2.0$ & $23.1(11.1-39.3)$ & $98.7(95.5-99.9)$ & $0.61(0.54-0.68)$ & $23.2(13.9-34.9)$ & $91.9(83.2-97.0)$ & $0.58(0.49-0.66)$ \\
\hline \multirow[t]{2}{*}{$\geq 3.25$} & $15.4(5.9-30.5)^{\mathrm{a}}$ & $100.0(97.7-100.0)$ & $0.58(0.50-0.65)$ & $5.8(1.6-14.2)^{\mathrm{a}}$ & $97.3(90.6-99.7)$ & $0.52(0.43-0.60)$ \\
\hline & \multicolumn{3}{|c|}{ Age-Platelet Index (API) } & \multicolumn{3}{|c|}{ Age-Platelet Index (API) } \\
\hline$>4$ & $37.5(22.7-54.2)$ & $94.8(90.3-97.6)$ & $0.66(0.59-0.72)$ & $15.6(7.7-26.9)$ & $93.8(86.0-97.9)$ & $0.55(0.46-0.63)$ \\
\hline$>5$ & $22.5(10.8-38.5)$ & $97.1(93.4-99.1)$ & $0.60(0.53-0.66)$ & $16.4(8.8-26.9)$ & $96.3(89.4-99.2)$ & $0.56(0.48-0.64)$ \\
\hline$>6$ & $15.0(5.7-29.8)^{a}$ & $100.0(97.9-100.0)$ & $0.58(0.51-0.64)$ & $8.2(3.0-17.0)^{a}$ & $98.8(93.2-99.9)$ & $0.53(0.45-0.59)$ \\
\hline$>7$ & $7.5(1.6-20.4)^{a}$ & $100.0(97.9-100.0)$ & $0.54(0.47-0.61)$ & $2.7(0.3-9.6)^{\mathrm{a}}$ & $98.8(93.2-99.9)$ & $0.51(0.43-0.59$ \\
\hline
\end{tabular}

AST aspartate aminotransferase, ALT alanine aminotransferase ratio, HBV Hepatitis B virus, AUROC Area under the receiver-operating characteristic. *numbers low in cells, analysis to be interpreted with caution
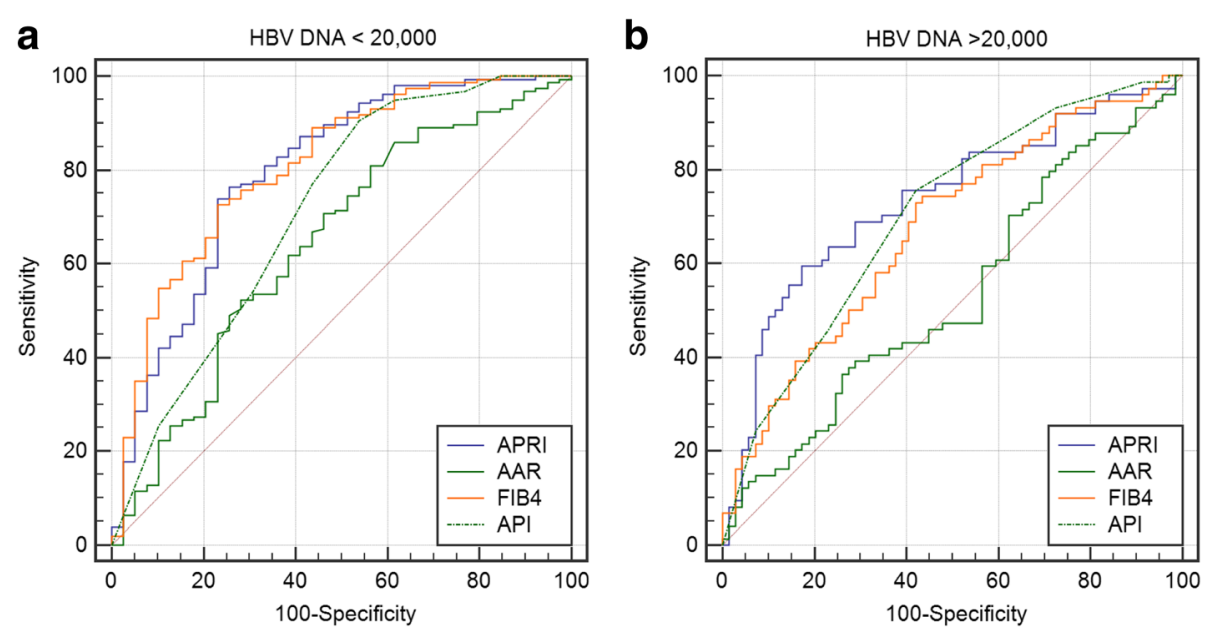

Fig. 1 a Receiver operating characteristics curve of the best compromise sensitivity-specificity of AST (aspartate aminotransferase)-platelet ratio index (APRI), AST/alanine aminotransferase ratio (AAR), FIB-4 index and age-platelet index (API) for identifying fibrosis score F2-4 in low-replicative HBV patients. In patients meeting these cut-offs, the sensitivity for APRI ( $\geq 0.33$ ) is $76.4 \%$ and the specificity is $74.4 \%$ (AUROC, 0.80; $95 \%$ Cl: $0.73-0.85$; $P<0.0001$ ), for AAR ( $\geq 0.93$ ) the sensitivity is $73.5 \%$ and the specificity $47.5 \%$ (AUROC, $0.62 ; 95 \%$ Cl: $0.55-0.69 ; P=0.023$ ), for FIB-4 ( $\geq 0.70$ ) the sensitivity is $72.9 \%$ and specificity is $76.9 \%$ (AUROC, $0.81 ; 95 \% \mathrm{Cl}: 0.75-0.86 ; P<0.0001$ ), and for API ( $>2$ ) the sensitivity is $75.1 \%$ and specificity is $55.0 \%$ (AUROC, $0.71 ; 95 \% \mathrm{Cl}: 0.64-0.77 ; P<0.0001)$. b Receiver operating characteristics curve of the best compromise sensitivity-specificity of AST (aspartate aminotransferase)-platelet ratio index (APRI), AST/alanine aminotransferase ratio (AAR), FIB-4 index and age-platelet index (API) for identifying fibrosis score F2-4 in high-replicative HBV patients. In patients meeting these cut-offs, the sensitivity for APRI ( $\geq 0.33)$ is $52.7 \%$ and the specificity is 87.0\% (AUROC, 0.73; 95\% Cl: $0.65-0.80 ; P<0.0001$ ), for AAR ( $\geq 0.91$ ) the sensitivity is $24.7 \%$ and the specificity $79.2 \%$ (AUROC, $0.52 ; 95 \%$ Cl: $0.44-0.60$; $P=0.643$ ), for FIB-4 $(\geq 0.70)$ the sensitivity is $58.1 \%$ and specificity is $66.7 \%$ (AUROC, $0.67 ; 95 \% \mathrm{Cl}: 0.59-0.75 ; P=0.0001$ ), and for API $(>2)$ the sensitivity is $75.0 \%$ and specificity is $56.2 \%$ (AUROC, $0.69 ; 95 \%$ Cl: $0.61-0.76 ; P<0.0001$ ) 


\section{Discussion}

Among the various markers available for evaluating HBV-related fibrosis, the ones utilized in this analysis are the most commonly studied, albeit not specifically in the context of low viremia levels. We have shown that APRI, FIB-4, AAR and API frequently identify F2-4 fibrosis, regardless of the HBV DNA levels. These findings are in concurrence with other studies dealing with chronic HBV in general that show a moderate utility in distinguishing F2-4 from F0-1 fibrosis. However, our analysis also demonstrates significant differences in the detection ability between patients with low and highreplicative states. Clearly, APRI seems to perform better in high-replicative patients (with only the cut-off $\geq 0.5$ reaching statistical significance). However, the other biomarkers appear to perform better in patients with low-replicative states, detecting fibrosis more frequently than in those with high replication.

According to our analysis, at their lowest conventional cut-off points, the AUROCs of all the biomarkers were of low diagnostic value, with only APRI (AUROC 0.62) reaching statistical significance. At a cut-off point of 0.5 , the higher sensitivity of APRI in high-replicative states was offset by a low specificity (69\%), while those with low replication exhibited a high specificity of $98 \%$. Similar results were seen in the sensitivity and specificity profile of the other biomarkers. However, despite these higher specificities, the overall accuracy of these biomarkers in diagnosing fibrosis remains poor in both groups of patients $(<0.70$ for all biomarkers at all conventional cut-offs), being far below the standard of diagnostic relevance (typically $>0.80$ ).

Interestingly, AAR appears better at distinguishing fibrosis in those with low replication. However, overall AAR only detects a quarter of the patients with F2-4 fibrosis with a diagnostic accuracy of 0.54 . Thus, its poor performance as a predictor of fibrosis makes it unsuitable for use in clinical settings. Moreover, these observations can also be extended to FIB-4 and API where significant fibrosis was missed in more than two-thirds of the overall cohort of patients at their lowest cut-offs, thereby diminishing the clinical relevance of these findings. Nonetheless, when analysing patients at ROCderived optimal cut-offs, all four biomarkers performed consistently better in the low-replicative state, suggesting greater diagnostic utility of these biomarkers in this category of patients. Our analysis shows that by lowering the cut-offs for APRI and FIB-4 a high diagnostic accuracy $(>0.80)$ may be achieved in distinguishing F2 -4 from F0-1 fibrosis in patients with low-replicative HBV.

The sensitivity levels of the biomarkers in identifying fibrosis in our study are somewhat lower (i.e. high number of patients with fibrosis incorrectly identified as being without) than that seen in other studies. In a recent meta-analysis [6], the summary sensitivities of APRI at cut-off levels of $0.5,0.7,1.0$ and 1.5 in identifying F2-4 fibrosis were $70,63.5,40$ and $34 \%$, respectively, compared to the sensitivities in our analysis of $62,43,24$ and $10 \%$, respectively. Similar differences were also seen in the summary sensitivities of FIB-4. These differences must be seen in the context of the patient population being studied, where in previous studies the HBV population was mostly comprised of patients with high viral replication and elevated ALT levels. In contrast, our study population bears a close resemblance to real-life $\mathrm{HBeAg}$-negative presentation, where a large number of patients had low replication and/or normal ALT. These consecutive patients also included those with 'high' replication and normal ALT, and 'low' replication with elevated ALT, eventually serving as a robust representative sample of HBeAg-negative patients. Thus, it is likely that the sensitivities and specificities shown in our analysis are closer to a real-life population, and the findings more representative.

The impact of elevated ALT and AST in our cohort could be more confounding than is apparent at face value. In most studies, patients with HBV who undergo liver biopsy are those that have high viral replication. However, in a more natural selection of HBV infected population, as in our cohort, liver enzyme elevation may be related to nonHBV factors such as steatosis, which in turn does not impact on development of fibrosis $[4,9,10]$. As such, this elevation of ALT and AST levels may cause an "artefactual" increase in APRI, FIB-4 and AAR thereby increasing the false-positive rate (i.e. high number of patients without fibrosis incorrectly identified as having disease).

Our analysis must be viewed in the context of its limitations. First, although we relied on pre-biopsy ALT and AST levels, it has been shown that HBV patients may show enzyme fluctuations [3,11, 12], and consequently some of these patients could feasibly be underscored, having dropped or increased the enzyme levels at the time of biopsy. Similarly, HBV DNA levels may also fluctuate over time, although fluctuations at $20,000 \mathrm{IU} / \mathrm{mL}$ are uncommon [13, 14]. In order to correct for such aberrations we categorized patients on the basis of the HBV DNA level recordings falling predominantly within the high or low replicative state. Overall, despite guidelinestratified thresholds, it remains a questionable approach to divide patients arbitrarily into low and high replicative states given the nature of HBV DNA fluctuations. Second, test accuracy can be ascertained when there is no selection bias in evaluating the reported outcome. Our population of HBV patients does not essentially represent an unselected cohort, given the impracticality of performing a liver biopsy in all HBeAg-negative patients. It is feasible that a proportion of these patients may have been biopsied as a consequence of subtle hints for the presence of 
fibrosis, particularly since patients who are not biopsied tend to have lower ALT and HBV DNA levels, thus escaping the trigger for a liver biopsy [14]. Clearly, these results must be validated in larger, unselected and properly stratified HBVinfected populations before generalizing these results. Despite these limitations, our study with a substantially large number of patients with low viral replication, offers a leading insight into a poorly appreciated disease state.

\section{Conclusion}

In conclusion, our study shows a modest accuracy of commonly used biomarkers in the diagnosis of significant fibrosis. In low-replicative patients, at conventional cut-offs, APRI is less useful while AAR is more useful in detecting fibrosis as compared to high replication patients, although it detects only a small minority of patients with fibrosis. Lowering the cut-offs may increase the diagnostic relevance of these biomarkers, particularly for APRI and FIB-4 in low-replicative disease.

\section{Additional files}

Additional file 1: Table S1. Comparison of the 4 biomarkers scores at various thresholds in low and high viremia levels between patients with minimal/mild (F0-1) and moderate-severe (F2-4) fibrosis. (DOCX 18 kb)

Additional file 2: Table S2 Sensitivity, specificity, positive and negative likelihood ratios of the four biomarkers at different cut-off values in identifying significant fibrosis (F2-4) in the overall cohort $(n=366)$ of patients. (DOCX $16 \mathrm{~kb}$ )

\section{Abbreviations}

AFP: a-fetoprotein; ALT: Alanine aminotransferase; AST: Aspartate aminotransferase; BMl: Body mass index; HBeAg: Hepatitis B envelope antigen; HBsAg: Hepatitis B surface antigen; HBV: Hepatitis B virus; ULN: Upper limit of normal

\section{Acknowledgements}

Not applicable

\section{Funding}

No current funding sources for this study.

\section{Availability of data and materials}

Study data will not be shared in compliance with local country regulations and in accordance with existing institutional IRB approvals.

\section{Authors contributions \\ Drs. FMS, TF, MAB, BEK, KIB helped in study concept and design; HA1, KA1, KSA, $H A 2$, FB, KA2, AA, IHA, MAB, KIB were responsible for acquisition of data; KA1, HA1, KA2, FB, FMS analysed and interpreted data; FMS, KA2, AA drafted the manuscript; All authors contributed to the critical revision of the manuscript for important intellectual content; FMS performed the statistical analysis. All authors read and approved the final manuscript.}

\section{Ethics approval and consent to participate}

The institutional review boards of all centres approved the study. Patient consent was not obtained, as this was a retrospective chart review.

\section{Consent for publication}

Not applicable

\section{Publisher's Note}

Springer Nature remains neutral with regard to jurisdictional claims in published maps and institutional affiliations.

\section{Author details}

${ }^{1}$ Liver Disease Research Center, King Saud University, Riyadh, Saudi Arabia. ${ }^{2}$ Department of Gastroenterology, Prince Sultan Military Medical City, Riyadh, Saudi Arabia. ${ }^{3}$ Division of Gastroenterology, Department of Medicine, King Fahad Hospital, Jeddah, Saudi Arabia. ${ }^{4}$ Division of Gastroenterology, Department of Medicine, King Faisal Specialist Hospital \& Research Center, Riyadh, Saudi Arabia. ${ }^{5}$ Department of Pathology, King Faisal Specialist Hospital \& Research Center, Riyadh, Saudi Arabia. 'Department of Pathology, King Abdulaziz Medical City, Riyadh, Saudi Arabia. ${ }^{7}$ Hepatobiliary Sciences \& Liver Transplantation, King Abdulaziz Medical City, Riyadh, Saudi Arabia. ${ }^{8}$ Gastroenterology Unit, Department of Medicine, King Abdulaziz Medical City, PO Box: 9515, Jeddah 21423, Saudi Arabia. ${ }^{9}$ King Saud bin Abdulaziz University for Health Sciences, Riyadh, Saudi Arabia. ${ }^{10}$ Gastroenterology Unit, Department of Medicine, King Fahad Armed Forces Hospital, Jeddah, Saudi Arabia.

Received: 4 March 2017 Accepted: 17 August 2017

Published online: 25 August 2017

References

1. European Association for the Study of the Liver. EASL clinical practice guidelines: management of chronic hepatitis B virus infection. J Hepatol. 2012;57:167-85.

2. Abaalkhail F, Elsiesy H, AlOmair A, Alghamdi MY, Alalwan A, AlMasri N. Et al; Saudi Association for the Study of liver diseases and transplantation (SASLT). SASLT practice guidelines for the management of hepatitis B virus. Saudi J Gastroenterol. 2014;20:5-25.

3. Iloeje $\mathrm{UH}$, Yang HI, Su J, et al. Risk evaluation of viral load elevation and associated liver disease/cancer-in HBV (the REVEAL-HBV) study group. Predicting cirrhosis risk based on the level of circulating hepatitis B viral load. Gastroenterology. 2006;130:678-86.

4. Abdo AA, Bzeizi Kl, Babatin MA, AlSohaibani F, AlMana H, Alsaad KO, et al. Predictors of significant fibrosis in chronic hepatitis $B$ patients with low viremia. J Clin Gastroenterol. 2014;48(6):e50-6.

5. Bravo AA, Sheth SG, Chopra S. Liver biopsy. N Engl J Med. 2001;344:495-500.

6. Xiao G, Yang J, Yan L. Comparison of diagnostic accuracy of aspartate aminotransferase to platelet ratio index and fibrosis-4 index for detecting liver fibrosis in adult patients with chronic hepatitis B virus infection: a systemic review and meta-analysis. Hepatology. 2015;61(1):292-302.

7. Saldanha J, Gerlich W, Lelie N, et al. An international collaborative study to establish a World Health Organization international standard for hepatitis B virus DNA nucleic acid amplification techniques. Vox Sang. 2001;80:63-71.

8. Intraobserver and interobserver variations in liver biopsy interpretation in patients with chronic hepatitis C. The French METAVIR Cooperative Study Group. Hepatology 1994;20:15-20.

9. Yun JW, Cho YK, Park JH, et al. Hepatic steatosis and fibrosis in young men with treatment-naïve chronic hepatitis B. Liver Int. 2009;29(6):878-83.

10. Shi JP, Fan JG, Wu R, et al. Prevalence and risk factors of hepatic steatosis and its impact on liver injury in Chinese patients with chronic hepatitis B infection. J Gastroenterol Hepatol. 2008;23(9):1419-25.

11. Papatheodoridis GV, Manesis EK, Manolakopoulos S, et al. Is there a meaningful serum hepatitis B virus DNA cutoff level for therapeutic decisions in hepatitis B e antigen-negative chronic hepatitis B virus infection? Hepatology. 2008:48:1451-9.

12. Kumar M, Sarin SK, Hissar S, et al. Virologic and histologic features of chronic hepatitis B virus-infected asymptomatic patients with persistently normal ALT. Gastroenterology. 2008;134:1376-84.

13. Papatheodoridis GV, Chrysanthos N, Hadziyannis E, et al. Longitudinal changes in serum HBV DNA levels and predictors of progression during the natural course of HBeAg-negative chronic hepatitis B virus infection. J Viral Hepat. 2008;15:434-41.

14. Sanai FM, Babatin MA, Bzeizi Kl, Alsohaibani F, Al-Hamoudi W, Alsaad KO, et al. Accuracy of international guidelines for identifying significant fibrosis in hepatitis B e antigen-negative patients with chronic hepatitis. Clin Gastroenterol Hepatol 2013;11:1493-99.e2. 